\title{
Measurement of the Complex Time-Frequency Channel Correlation Function
}

\author{
Phillip A. Bello \\ Contribution From ADCOM, Inc., 808 Memorial Drive, Cambridge 39, Mass.
}

(Received March 20, 1964)

\begin{abstract}
The time and frequency selective fading properties of radio channels may be characterized by evaluating the cross-correlation function between two received carriers as a function of their frequency separation. In practice such correlation functions have been measured using only the envelopes of the received carriers. Recent studies of the effect of time and frequency selective fading on digital data transmission have shown that envelope correlation information is insufficient for an accurate evaluation of system performance and that the complex envelope correlation function is needed. This paper presents an experimental technique for the measurement of the complex time-frequency correlation function of radio channels that uses independent frequency standards at transmitter and receiver. An analytical study is made of the theory of operation of this system and of the effects of instabilities of the frequency standards.
\end{abstract}

The transmission of a sinusoid through a fading radio channel results in the reception of a narrowband random process. Let $x(t)$ denote the received narrow band waveform resulting from the transmission of a sinusoid $f \mathrm{c} / \mathrm{s}$ away from a "carrier" frequency of $f_{\mathrm{o}} \mathrm{c} / \mathrm{s}$. Then $x(t)$ may be expressed as

$$
x(t)=\operatorname{Re}\left\{\boldsymbol{T}(f, t) e^{j 2 \pi\left(f_{0}+f\right) t}\right\}
$$

where $T(f, t)$, the complex envelope of the received narrow band process, is the Time Variant Transfer Function of the medium when measured with respect to the carrier frequency $f_{0}$.

The correlation properties of this transfer function are defined by the correlation function

$$
R_{f, t}(\tau, \Omega)=\overline{T^{*}(f, t) T(f+\Omega, t+\tau)}
$$

where * denotes the complex conjugate and the overline indicates an ensemble average. Knowledge of the channel corre'ation function $R_{f, t}(\tau, \Omega)$ is essential to determining the degradation of communication system performance caused by the time and frequency selective fading of the channel.

As defined in (2), the channel correlation function involves an ensemble average. Unfortunately, an ensemble of channels is not available and thus the ensemble averages may not be evaluated experimentally as averages over an ensemble of channels. In practice, the ensemble of interest is generated by considering the behavior of a single channel at a set of mutually exclusive time intervals of the same duration. In this way the behavior in a given time interval provides the typical member function of the ensemble, and ensemble averages are interpreted as appropriate time averages.
The measurement problem is complicated by the fact that physical channels do not exhibit statistical regularity over indefinitely long intervals of time. Thus there is an inherent conflict between the desire to have a long measurement time in order to reduce measurement errors due to finite sample size and the desire to have a short enough measurement time so that the desired statistical parameters have changed negligibly due to the instability of the statistical structure of the channel.

Bello [1963] has derived the quasi-WSSUS channel to account for the fading characteristics observed on many radio channels. A WSSUS (Wide Sense Stationary Uncorrelated Scattering) channel is one which, for analytical purposes, may be represented as a continuum of uncorrelated scatterers fluctuating with wide sense stationary statistics. A necessary and sufficient condition for a channel to be WSSUS is that the channel correlation function $R_{f}, t(\tau, \Omega)$ be independent of $f, t$; i.e., independent of the location of the time interval and the frequency band within which the measurement of the correlation function was made. Thus

$$
R_{f},{ }_{t}(\tau, \Omega)=R(\tau, \Omega)
$$

for the WSSUS channel.

The quasi-WSSUS or QWSSUS channel is one in which $R_{f},{ }_{t}(\tau, \Omega)$ changes sufficiently "slowly" with changes in $f$ and $t$. In particular, $R_{f}, t(\tau, \Omega)$ is assumed to change negligibly for values of $f, t$ in a time-frequency rectangle whose time dimension is equal to the input signaling element duration plus the multipath spread of the channel and whose frequency dimension is equal the bandwidth of the signaling element.

1 The work reported here was performed under subcontract to ITT Communications Systems, Inc., Paramus, N.J. 
The utility of the QWSSUS channel concept is that, when applicable, it allows one to represent the channel as a WSSUS channel, at least as far as its effect upon any particular signaling element is concerned.

Most radio channels exhibit a "fast" fading (e.g., a few c/s) superimposed upon a "slow" fading (e.g., hourly or diurnal variations). In order to measure the channel correlation functions by time averages the measurement time $T_{m}$ must satisfy the inequality

$$
T_{m}<<\frac{1}{\theta_{\max }}
$$

where $\theta_{\max }$ is the bandwidth of the slow fluctuations. On the other hand, to keep the measurement errors due to finite sample size small one must have

$$
T_{m}>>\frac{1}{B_{f}}
$$

where $B_{f}$ is the bandwidth of the fast fluctuations. It follows that the short-time correlation functions can be measured only if the bandwidth of the fast fluctuations is a few orders of magnitude greater than the slow fluctuations. Fortunately, it appears that many radio channels exhibit this property. The following discussion assumes that (4) and (5) are satisfied and that over the measurement interval we can represent the channel by a hypothetical WSSUS channel.

One further restriction needs to be imposed on the channel if the measurement techniques to be described are to succeed. This restriction is that the "spread factor" of the channel be less than unity, where the spread factor, $k$, is given by the ratio

$$
k=\frac{B_{f}}{B_{c}}
$$

in which $B_{c}$ is the correlation bandwidth of the channel being measured. The reason for this restriction will become evident during the subsequent discussion. Fortunately, except for certain esoteric radio channels (e.g., the orbital dipole channel), the spread factor is less than unity and thus the measurement technique to be described is quite generally useful.

We consider here the direct measurement of the correlation function $R_{f^{\prime}, t^{\prime}}(\Omega, \tau)$, or rather $R_{0, t^{\prime}}(\Omega, \tau)$, since it is assumed that the center frequency $f_{0}$ is chosen coincident with the "center" frequency of signaling waveforms to be transmitted. The basic measurement technique involves the transmission of two sinusoids separated by a frequency of $\Omega \mathrm{c} / \mathrm{s}$ and the determination of the cross-correlation function between the complex envelopes of the two received processes. Such correlation functions have never been measured for radio channels. The nearest approximation to this type of measurement is that performed by Claxton and Anderson [1962] in a lunar reflection study. They generated the two sinusoids as sidebands on an amplitude modulated carrier and determined the cross-correlation function between the envelopes (the magnitudes of the complex envelopes) of the two received processes. Thus, in effect, what they measured is given by

$$
\tilde{R}_{0, t^{\prime}}(\Omega, \tau) \equiv \overline{\left.T^{*}\left(-\Omega / 2, t^{\prime}-\tau / 2\right)\right|_{i} T\left(\Omega / 2, t^{\prime}+\tau / 2\right) \mid}
$$

rather than

$$
R_{0, t^{\prime}}(\Omega, \tau)=\overline{T^{*}\left(-\Omega / 2, t^{\prime}-\tau / 2\right) T\left(\Omega / 2, t^{\prime}+\tau / 2\right)} .
$$

While the correlation function (7) is considerably easier to measure, it does not contain information due to decorrelation in the phase of the transfer function. Moreover, if the channel is indeed gaussian and QWSSUS, (7) does not provide sufficient statistical information for a complete characterization of the channel. Thus, for example, the error probability and intermodulation distortion calculations performed by Bello and Nelin [1964a, b] cannot be carried through unless (8) rather than (7) is available.

The measurement of (8) is difficult, not only because it requires that two narrowband processes at different center frequencies be manipulated so as not to introduce relative frequency instabilities, but also because it requires at the transmitter the generation of two sinusoids of different frequency with sufficiently small relative frequency instability.

In figure 1 we present the block diagram of a measurement method which appears capable of measuring $R(\Omega, \tau)$ for a range of situations of practical interest. The success of the method depends upon the use of sufficiently stable clocks in the transmitter and receiver. If clocks of the required stability are not available, a common timing source must be provided. Two methods for achieving a common timing source are the use of WWVL transmissions and the use of a landline to provide the receiver with transmitter clock signals.

We shall now discuss in some detail the theory of operation of the measurement technique of figure 1 . A frequency standard in the transmitter whose output is nominally at $F \mathrm{c} / \mathrm{s}$ is used in combination with a frequency synthesizer to g'enerate a group of very stable sinusoids separated by multiples of $F \mathrm{c} / \mathrm{s}^{2}$ This frequency group is translated to an appropriate RF frequency $f_{0}$ by heterodyning operations and then transmitted. Two types of frequency instabilities are inescapably associated with the transmitted output, namely, differential instability due to instability of the frequency standard and common instability due to instabilities in the heterodyning oscillators. From a mathematical point of view, common instabilities in the transmitter and receiver heterodyning oscillators may be regarded as part of the channel since they are also present when the channel is used for communication purposes. Thus common instabilities are not harmful provided they are small compared to the instability introduced by

${ }^{2}$ In practice it may be desirable to generate this frequency group by frequency modulating an oscillator with the frequency standard output and a modulation index chosen to given sidebands and carriers of not too different amplitudes. 


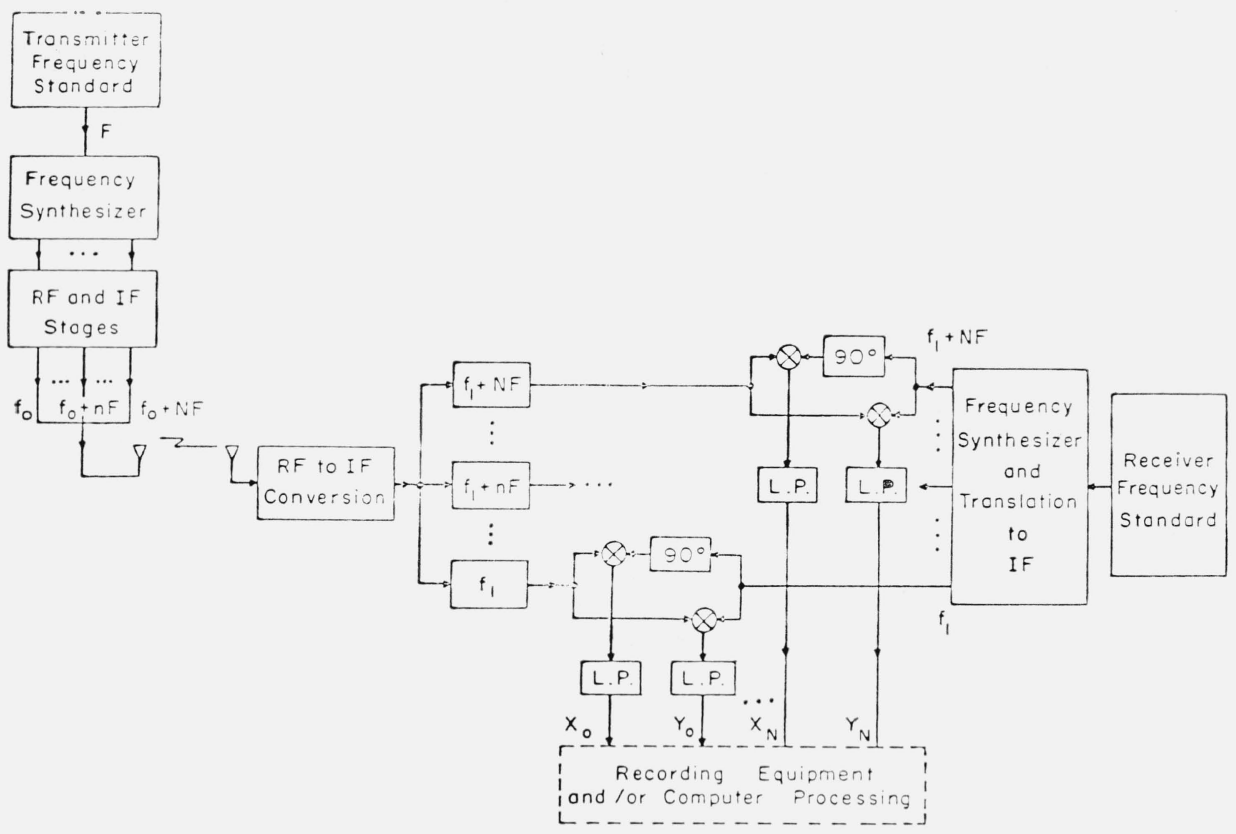

Figure 1. Block diagram of complex correlation function measurement method.

the medium itself - a situation readily achieved in practice. On the other hand, differential instabilities are quite harmful since they are falsely interpreted by the measurement equipment as a frequency selective behavior. Moreover, this frequency selective behavior caused by differential frequency instability, in contrast to the effects of common frequency instability, are not present when the channel is used for communication.

From the above observations of equipment frequency instability, we conclude that in our analysis of the measurement technique of figure 1, it is sufficient to neglect the common frequency instabilities introduced in the transmitter and receiver, assuming that they have been included as part of the transmission medium. Moreover, as may readily be appreciated, it is only the relative instability between the transmitter and receiver frequency standards that enters in the accuracy of the measurement technique, so that for analytical purposes it is sufficient to assume a perfect frequency standard at the receiver and a hypothetical frequency standard at the transmitter whose frequency instability is that observed for the relative frequency of two independent frequency standards.

In view of the above, the transmitter output $y(t)$ may be expressed as

$$
y(t)=\sum_{n=0}^{N} \cos \left[2 \pi\left(f_{0}+n F\right) t+n \delta F t+n \phi(t)\right]
$$

where $f_{\mathrm{o}}$ is the RF frequency, $\delta$ is a random variable describing the long term percentage drift between two independent frequency standards, and $\phi(t)$ describes the short term phase fluctuations about the long term phase drift $\delta F t$. An initial analysis was carried through, including the phase fluctuation term $\phi(t)$. However, it was found to produce negligible measurement error compared to the long term phase drift $\delta F t$. Thus, to simplify our discussion we will neglect the short term phase fluctuations and use

$$
y(t)=\sum_{n=0}^{N} \cos \left[2 \pi\left(f_{0}+n F\right) t+n \delta F t+n \theta\right]
$$

in place of (9), where $\theta$ is a constant denoting a random initial phase at $t=0$.

According to our definition of $T(f, t)$ we see that if $y(t)$ in $(10)$ is the transmitted process; then, after translation to an IF frequency of $f_{1} \mathrm{c} / \mathrm{s}$, the received process complex envelope is given by

$$
w(t)=\sum_{n=0}^{N} T(n F, t) e^{j 2 \pi\left(f_{1}+n F+n F \delta\right) t+n \theta} .
$$

To be exact one should use $T(n F+n F \delta, t)$ in the summation in (11) rather than $T(n F, t)$. However, for any physical channel these will be indistinguishable since $\delta$ will be extremely small $\left(10^{-10}\right.$ perhaps $)$.

Each of the $N+1$ fading carriers at the frequencies $f_{1}+n F ; n=0, \ldots, N$ is extracted by means of a filter bank. Some attention must be given to the 
design of these filters so that, with respect to their center frequencies, their amplitude and phase characteristics are essentially identical over the bandwidth of the input fading carrier. This should not be a very difficult problem since for virtually all channels of interest the required value of $F$ to sample the channel correlation function accurately will be much larger than the fading bandwidth, and thus each filter bandwidth can be much wider than the bandwidth of the process it is desired to filter.

At the receiver a frequency standard and synthesizer generates a set of sinusoidal components at the nominal frequencies $f_{1}+n F ; n=0, \ldots N$. As discussed above, we have analytically taken account of receiver frequency standard instabilities by including them in the transmitter frequency standard instability and heterodyning oscillator instabilities by including them in the channel. Thus the output of the receiver frequency synthesizer in figure 1 may be represented as the set of discrete frequency components $\cos 2 \pi\left(f_{1}+n F\right) t ; n=0, \ldots . . N$. With the aid of $90^{\circ}$ phase shifters, the set of components sin $2 \pi\left(f_{1}+n F\right) t ; n=0, \ldots, N$ is also generated, and then coherent detection of the in-phase and quadrature components of the various received carriers is performed. The bandwidths of the low-pass filters following the multipliers are just wide enough to pass the fluctuations due to the fading of the corresponding carrier. It is readily seen that the outputs $X_{n}(t)$ and $Y_{n}(t)$ in figure 1 are given by

$$
\begin{aligned}
& X_{n}(t)=\operatorname{Re}\left\{T(n F, t) e^{j[2 \pi n F \delta t+n \theta]}\right\} \\
& Y_{n}(t)=\operatorname{Im}\left\{T(n F, t) e^{j[2 \pi n F \delta t+n \theta]}\right\} .
\end{aligned}
$$

It will now be demonstrated that by appropriate processing of the set of waveforms $\left\{X_{n}(t), Y_{n}(t)\right\}$ it is possible to determine the required channel correlation function. The set of waveforms $\left\{X_{n}(t), Y_{n}(t)\right\}$ may be recorded on tape or film and later processed by a digital computer or else processed in real time with an analog computer.

As discussed previously, we shall assume that over the measurement period we may assume the channel to be WSSUS. Thus the correlation function

$$
\begin{aligned}
& \overline{T^{*}(f, t) T(f+\Omega, t+\tau)} \\
& =\left\langle T^{*}(f, t) T(f+\Omega, t+\tau)\right\rangle=R(\Omega, \tau) .
\end{aligned}
$$

If we form the functions $X_{0 n}(t), Y_{0 n}(t)$ given by

$$
\begin{aligned}
& X_{0 n}(t)=X_{0}(t) X_{n}\left(t+\tau_{1}\right)-Y_{0}(t) Y_{n}\left(t+\tau_{1}\right) \\
& Y_{0 n}(t)=X_{0}(t) Y_{n}\left(t+\tau_{1}\right)+Y_{0}(t) X_{n}\left(t+\tau_{1}\right)
\end{aligned}
$$

we readily determine from (24) that

$$
\begin{aligned}
& X_{0 n}(t)=\operatorname{Re}\left\{T^{*}(0, t) T\left(n F, t+\tau_{1}\right) e^{j[2 \pi n \delta F t+n \theta]}\right\} \\
& Y_{0 n}(t)=\operatorname{Im}\left\{T^{*}(0, t) T\left(n F, t+\tau_{1}\right) e^{j[2 \pi n \delta F t+n \theta]}\right\}
\end{aligned}
$$

where $\theta$ in (15) differs from $\theta$ in (12) in containing an additive term $2 \pi n \delta F \tau_{1}$. By performing the short time averages

$$
\begin{aligned}
\left\langle X_{0 n}(t)\right\rangle_{T_{m}} & =\frac{1}{T_{m}} \int_{t-T_{m} / 2}^{t+T_{m} / 2} X_{0 n}(s) d s \\
\left\langle Y_{0 n}(t)\right\rangle_{T_{m}} & =\frac{1}{T_{m}} \int_{t-T_{m} / 2}^{t+T_{m} / 2} Y_{0 n}(s) d s
\end{aligned}
$$

we can determine real and imaginary parts of

$$
\begin{aligned}
Z_{0 n}(t) & =\frac{e^{j n \theta}}{T_{m}} \int_{t-T_{m} / 2}^{t+T_{m} / 2} T^{*}(0, s) T\left(n F, s+\tau_{1}\right) e^{j 2 \pi n \delta F s} d s \\
& =\frac{e^{j n \theta}}{T_{m}} \int_{-T_{m} / 2}^{T_{m} / 2} T^{*}(0, s-t) T\left(n F, s-t+\tau_{1}\right) e^{j 2 \pi n \delta F s} d s
\end{aligned}
$$

where $\theta$ in the second equation in (17) differs from the first equation by the quantity $-2 \pi n \delta F T_{m}$.

Now the product $T^{*}(0, s-t) T(n F, s-t+\tau)$ contains a direct current value plus a fluctuating component; i.e.,

$$
T^{*}(0, s-t) T\left(n F, s-t+\tau_{1}\right)=R\left(n F, \tau_{1}\right)+f_{n}(t)
$$

and it is the purpose of the time average to average out the fluctuations $f_{n}(t)$. Assuming that $T_{m}$ is large enough to perform this averaging we find that

$$
\begin{aligned}
Z_{0 n}(t) & =e^{j n \theta}\left\langle\left[R\left(n F, \tau_{1}\right)+f_{n}(t)\right] e^{j 2 \pi n \delta F t}\right\rangle_{T_{m}} \\
& \approx e^{j n \theta} R\left(n F, \tau_{1}\right)\left\langle e^{j 2 \pi n \delta F t}\right\rangle_{T_{m}} \\
& =e^{j n \theta} \operatorname{sinc}\left[n F \delta T_{m}\right] R\left(n F, \tau_{1}\right)
\end{aligned}
$$

where,

$$
\operatorname{sinc} x=\frac{\sin \pi x}{\pi x}=1-\frac{(\pi x)^{2}}{3 !}+\frac{(\pi x)^{4}}{5 !} \cdots
$$

From (19) we note that

$$
\begin{aligned}
\left|Z_{0 n}\right| & =\left|\operatorname{sinc} n F \delta T_{m}\right|\left|R\left(n F, \tau_{1}\right)\right| \\
\Varangle Z_{0 n} & =n \theta+\Varangle R\left(n F, \tau_{1}\right) .
\end{aligned}
$$

Thus the clock frequency instabilities appear as an error only in the measurement of the magnitude of the required channel correlation function while the ambiguity in initial phase affects only the angle of the channcl correlation function. The greatest error in the magnitude occurs at $n=N$. It is probably sufficient for most purposes to require

$$
1-\left|\operatorname{sinc} N F \delta T_{m}\right| \leq 0.1
$$

then, from the expansion in (20)

$$
N F \delta T_{m}<0.25 \text {. }
$$


To obtain an idea of the long term stability required in a rather extreme case assume

$$
\begin{aligned}
& N F=10 \text { megacycles per second } \\
& T_{m}=100 \text { seconds, }
\end{aligned}
$$

Then from (23) we find that

$$
\delta<2.5 \times 10^{-10}
$$

which is just within the capability of crystal controlled frequency standards.

We will now describe a method to remove the effects of the angle $\theta$. Unfortunately this method not only removes the effect of the angle $\theta$, but it removes the effect of any linear variation of $\Varangle R(\Omega, \tau)$ with $\Omega$. This fact becomes obvious when we write $n \theta$ in the form

$$
n \theta=n 2 \pi F T_{1}
$$

where

$$
T_{1}=\frac{\theta}{2 \pi F}
$$

It is readily demonstrated that a linear variation of $\Varangle R(\Omega, \tau)$ with $\Omega$ of the form $2 \pi \Omega \tau_{0}$ is directly interpretable as the existence of a mean path delay $\tau_{0}$. Thus, from (26) we see that the presence of the angle $n \theta$ may be interpreted physically as adding to the channel an unknown path delay $T_{1}$.

For purposes of discussion, assume that any mean path delay has been absorbed by $T_{1}$ so that $\Varangle R(\Omega, \tau)$ contains no linear variation with $\Omega$. In actuality the phase of $Z_{0 n}$ will be measured only in a range of $2 \pi$, say from $-\pi$ to $\pi$. Thus let

$$
\phi_{n}=\left\{\Varangle Z_{0 n}\right\} \text { Modulo } 2 \pi
$$

be the angle actually measured for $\Varangle Z_{0 n}$. Form the second order difference

$$
\Delta^{2} \phi_{n}=\left\{\left[\phi_{n}-\phi_{n-1}\right]-\left[\phi_{n-1}-\phi_{n-2}\right]\right\} \text { Modulo } 2 \pi .
$$

Then it is readily demonstrated that

$$
\Delta^{2} \phi_{n}=\Delta^{2}\left[\Varangle R\left(n F, \tau_{1}\right)\right] \operatorname{Mod} 2 \pi .
$$

If the procedure is now reversed and it is assumed that $\Varangle R\left(\Omega, \tau_{1}\right)$ varies small enough in an $\Omega$ interval of duration $F$ so that

$$
\begin{aligned}
\Delta\left[\Varangle R\left(n F, \tau_{1}\right)\right]= & \Varangle R\left(n F, \tau_{1}\right) \\
& \quad-\Varangle R\left([n-1] F, \tau_{1}\right)<<2 \pi
\end{aligned}
$$

one finds that the resulting set of points will form a smooth curve except where it reaches the limits $-\pi$ or $\pi$. These points of discontinuity may be removed by adding or subtracting $2 \pi$ and continuing the curve smoothly. Presuming this has been done, the resulting curve will differ from the actual $\Varangle R\left(\Omega, \tau_{1}\right)$ only in a linear variation of the form $2 \pi \Omega T_{1}$ and in a constant value which is equal to $\Varangle R\left(0, \tau_{1}\right)$. This initial angle may be found from $\Varangle Z_{00}$ since

$$
\Varangle Z_{00}=\Varangle R\left(0, \tau_{1}\right) .
$$

As a final note it should be pointed out that if one may assume that the channel has gaussian statistics it is possible to determine $\left|R\left(n F, \tau_{1}\right)\right|$ by an alternate procedure that is unaffected by the instability of the frequency standards. This method involves a determination of the set of correlations between envelopes of the outputs of filter bank in figure 1 ; i.e., a determination of

$$
W_{0 n}=\left\langle|T(0, t)|\left|T\left(n F, t+\tau_{1}\right)\right|\right\rangle T_{m} .
$$

If one assumes that $T(f, t)$ is normally distributed, then, assuming that $T_{m}$ is large enough to average out the fast channel fluctuations one may show from the properties of a gaussian process that

$$
\frac{W_{0 n}}{R(0,0)}=2 E\left[\rho\left(n F, \tau_{1}\right)\right]-\left[1-\rho\left(n F, \tau_{1}\right)^{2}\right] \mathrm{K}\left[\rho\left(n F, \tau_{1}\right)\right]
$$

where

$$
\rho\left(n F, \tau_{1}\right)=\left|\frac{R\left(n F, \tau_{1}\right)}{R(0,0)}\right|
$$

and $E[], K[]$ are complete elliptic integrals of the second and first kind, respectively. Because of the monotonic relationship between $W_{0 n} / R(0,0)$ and $\rho\left(n F, \tau_{1}\right)$, it is possible to determine $\left|R\left(n F, \tau_{1}\right)\right|$ once $W_{0 n}$ and $R(0,0)$ are measured.

\section{References}

Bello, P. A. (Dec. 1963), Characterization of randomly timevariant linear channels, IEEE Trans. Commun. Systems CS-11, No. 4, 360-393. (This paper is identical to ADCOM Research Report No. 2, Aug. 1962.)

Bello, P. A., and B. D. Nelin (March 1964a), The effect of frequency selective fading on intermodulation distortion and sub-carrier phase stability in frequency modulation systems, IEEE Trans. Commun. Systems, in press. (ADCOM, Inc. Research Report No. 5, March 15, 1963.)

Bello, P. A., and B. D. Nelin (March 1964b), The effect of combined time and frequency selective fading on the binary error probabilities of incoherent matched filter receivers, IEEE Trans. Commun. Systems, in press. (ADCOM, Inc. Research Report No. 7, March 1963).

Claxton, B. H., and R. E. Anderson (Aug. 31, 1962), Lunar reflection study, Final Report of the General Engineering Laboratory of General Electric Co., to Air Force Cambridge Research Lab., Contract No. AF 19(604)-6634.

(Paper 68D10-416) 